Recepción: 20 / 04 / 2017

Aceptación: 20 / 05 / 2017

Publicación: 15 / 07 / 2017
Ciencias de la computación Artículo Científico

\title{
Las interfaces de los sistemas de información robóticas
}

\author{
The interfaces of robotic information systems
}

\section{Interfaces sistemas de informação robóticos}

\author{
Michelle A. Varas-Chiquito ${ }^{\text {I }}$ \\ michelle.varasch@ug.edu.ec \\ Leslie León-Bassantes II \\ leslie.leonb@ug.edu.ec \\ Ulises Villacis-Chanca ${ }^{\text {III }}$ \\ ulises.villacisch@ug.edu.ec \\ María M. Jaramillo-Campos ${ }^{\text {IV }}$ \\ maria.jaramilloca@ug.edu.ec
}

Correspondencia: michelle.varasch@ug.edu.ec 


\section{Resumen}

Durante muchos años los sistemas de información en sus diferentes gamas han ayudado considerablemente a la toma de decisiones y al conocimiento total de la información que ella necesita replicar, logrando así las mejoras en la producción; siendo un conjunto de elementos para un fin común, brindando información importante de forma rápida, entendible, y sobre todo traducida a un lenguaje fácil de leer o visualizar, siendo estas interfaces también autónomas y sistematizadas. Este análisis es sustentado mediante una metodología de investigación de los diferentes proyectos y estudios realizados; los cuales se han convertido en artífices de la comunicación que expresan los dispositivos electrónicos especializados al cual llamaremos robóticos, en el presente artículo se muestra el análisis y utilidad de las herramientas que permiten el enlace y la vinculación logrando convertirse en interfaces visuales de los organismos robóticos, esta integración nos da como resultado y conclusión que la simbiosis de tecnología resulta una ayuda inmensa y eficiente sobre todo en el ahorro del tiempo, se recomienda el uso de estas interfaces agregando además las interfaces gráficas y maximizar su implementación en los diferentes aspectos de producción así como también en el ámbito educativo para integrar estas tecnologías de forma exponencial.

Palabras claves: Interfaces; información; sistemas; robótica; análisis. 


\section{Abstract}

For many years the information systems in their different ranges have helped considerably the decision making and the total knowledge of the information that it needs to replicate thus achieving improvements in production, being a set of elements for a common purpose, providing important information in a fast, understandable, and above all translated into a language easy to read or visualize these interfaces being also autonomous and systematized. This analysis is supported by a research methodology of the different projects and studies carried out; which have become communication important links expressing the specialized electronic devices which will be called robotics. The present article shows the analysis and utility of the tools that allow the linking and the linkage managing to become visual interfaces of the robotic organisms, this integration gives as a result and conclusion that the technology symbiosis is an immense and efficient aid especially in saving time, it is recommended the use of these interfaces adding also the graphical interfaces and maximize their implementation in the different aspects of production as well as in the educational field to integrate these technologies exponentially.

Keywords: Interfaces; information; systems; robotics; integration. 


\section{Resumo}

Por muitos anos os sistemas de informação em várias categorias que ajudaram consideravelmente a tomada de decisões e o pleno conhecimento das informações que ela precisa para replicar, conseguindo melhorias na produção; sendo um conjunto de elementos para um propósito comum, fornecendo informações importantes rapidamente, compreensível, e acima de tudo traduzido em uma linguagem fácil de ler ou entender, estes também ser interfaces de autônomos e sistematizadas. Esta análise é apoiado por uma metodologia de vários projectos e estudos de pesquisa; que eles se tornaram criadores de comunicação expressando dispositivos eletrônicos especializados que chamaremos robótico, nesta análise artigo e ferramentas de serviços públicos que permitem a ligação e articulação mostrada conseguiu tornar-se interfaces visuais organismos robóticos essa integração dá resultados e concluiu que a simbiose da tecnologia é uma ajuda enorme e eficiente, especialmente na economia de tempo, o uso dessas interfaces é recomendado também adicionando interfaces gráficas e maximizar a sua implementação nos diferentes aspectos da produção bem como na educação de integrar estas tecnologias de forma exponencial.

Palavras-chave: Interfaces; sistemas de informação; robótica; análise. 


\section{Introducción}

Análisis basado en la importancia, mejoras y algunas actualizaciones en la forma de mostrar los datos enviados por organismos robóticos a través de diferentes softwares que permiten leer estos datos de forma sencilla para diferentes tipos de usuarios desde niños, jóvenes estudiantes y expertos a los cuales les permite identificar los resultados ya sean estos esperados o no.

\section{Introducción al problema}

Los resultados de un proceso informático entregados ya sean por sistemas transaccionales y en nuestro caso de estudio elementos electrónicos, no son de fácil entendimiento o no permiten una toma de decisiones inmediata ya sea por la estructura misma de los datos como el tipo de software utilizado, incluyéndose también aspectos como la velocidad en la que se presenta esta información, y sobre todo en la forma que se presenta siendo esta no entendible ni amigable, y no permite la interacción del usuario con los datos, y en la mayoría de los casos en un proceso educativo donde estas interfaces hacen más fácil el estudio y el aprendizaje de la programación logrando una motivación a esta actividad, he ahí el conjunto de la importancia de las interfaces de información en los sistemas robóticos.

El desarrollo de estas interfaces se encuentra en constante evolución, empresas como lego con su producto LEGO MINDSTORMS NXT que han presentado nuevas formas de aprendizaje e interacción, en la actualidad son de gran beneficio para el aprendizaje de jóvenes en el mundo de la robótica.

La hipótesis planteada para este estudio converge en conocer si estas nuevas interfaces se convierten en elementos importantes y cuáles de estas características hacen que las mismas resalten para los usuarios, y a su vez, estos las prefieran gracias a la facilidad de entendimiento e interacción. La 
hipótesis planteada está relacionada con la investigación en base a estudios de algunas de estas herramientas sus características y nivel de aceptación.

\section{Importancia del problema}

Existen gastos en la adquisición de equipos de lectura de información, gastos sustantivos en contabilidad de las empresas, que en muchas ocasiones el usuario termina no solo malinterpretando estas lecturas sino teniendo cansancio mental; requiriendo personal especializado que las interpreten y en el caso de algunos centros de estudio, la planificación de la enseñanza es compleja para lograr de mejor manera el aprendizaje así como incentivar el estudio en los alumnos; muchos adolescentes terminan retirándose de las carreras tecnológicas por la falta de asimilación o interés en el aprendizaje de los diferentes lenguajes de programación, estas instituciones trabajan sin saber que algunas interfaces pueden influenciar en los mejores resultados de producción así como en los resultados de aprendizaje en el uso de herramientas tecnológicas, maximizando el rendimiento de todos aquellos que la interpretan y utilizan.

\section{Metodología}

Las metodologías de esta investigación están diseñadas con el objeto de reunir información variada que explique conceptos o eventos, están basadas en la recolección de información bibliográfica, así como de otros estudios que serán mostrados, plasmados y analizados en el presente artículo con el objetivo que los resultados permitan tomar decisiones de cambios y así futuras implementaciones de nuevos softwares incluso el desarrollo de los mismos. Estos métodos de recopilación de información mantendrán la objetividad de la investigación así como su diversidad de muestras. 


\section{Resultados}

Para este estudio partiremos con decir que la robótica en el área educativa utiliza como factor principal organismos electrónicos llamados robots como un recurso didáctico que permite a los alumnos construir su propio conocimiento de una forma práctica, visual y movilizadora, donde les permitirá fortalecer los frentes de innovación e investigación y en el ámbito profesional no solo de clientes consumidores agregando a este aspecto la otra parte esencial de nuestra investigación las interfaces amigables, es así que para hacer que los estudiantes maximicen su experiencias y maximicen su tiempo en estas investigaciones se hace imprescindible y fundamental el uso de las interfaces adecuadas y sobre todo mejoradas que permitan a los usuarios que reciben resultados de lecturas analógicas y textuales, usuarios sin experiencia, ajenos o con poco interés de aprender involucrándolos a interactuar con robots de manera sencilla, y divertida. Estos conceptos son fortalecidos con el desarrollo de ERBPI (Easy Robot Behaviour Programming Interface) una interface de programación de Robots móviles que no requiere que el usuario tenga experiencia previa en programación, sin embargo, para hacer realidad este tipo de interfaces sus constructores se apartaron del paradigma de programación imperativa y trabajaron más en los enfoques de comportamientos, dando al usuario lecturas y visualización de todos los elementos robóticos como son los actuadores, diferentes sensores que interactúan entre si y su posible comportamiento con su entorno. Este software ERBPI es reconocido por estar diseñado para trabajar con diferentes organismos robóticos integrando sus partes y las experiencias didácticas en cursos y talleres que les permitió maximizar la experiencia didáctica educativa.

Cuando se habla de interfaces robóticas ésta se integra con el concepto de robótica pedagógica que tiene como finalidad la de explotar el deseo de los usuarios por interactuar con un robot para favorecer los procesos cognitivos. Martial Vivet (Actes du Premier Congrès Francophone de 
Robotique Pédagogique Édités par Pierre Nonnon et Martial Vivet, Le Mans, Francia 24 au 27 août 1989.) propone la siguiente definición de robótica pedagógica:

Es la actividad de concepción, creación y puesta en funcionamiento, con fines pedagógicos, de objetos tecnológicos que son reproducciones reducidas muy fieles y significativas de los procesos y herramientas robóticas que son usados cotidianamente, sobre todo, en el medio industrial.

Martial Vivet

Además, la robótica como recurso educativo permite desarrollar de manera natural conocimientos de Ciencia y Tecnología en general. Muchos sistemas de información que priorizan las exigencias de la informática sobre las de los usuarios están destinados tal vez a fracasar y en algunos de los casos al olvido. Las grandes limitaciones actuales conocidas como interacción humano-computador, primordialmente en los usuarios que no gozan de interfaces disponibles para su labor, se originaron cuando el sistema fue desarrollado obviando gran colección de información como el diseño general, a la vez de obviar también pasos fundamentales como la participación y la identificación de las necesidades sobre todo de las expectativas del usuario de trabajar y conocer información vital de manera sencilla en un interfaz de lectura de información, es decir, no solo la integridad de los datos es vital sino la recepción e interpretación de los mismos.

En el desarrollo de sistemas de gestión de información creativos es bien cierto que surge la necesidad de conocer la complejidad interna de los elementos robóticos y sus características para que sean transmitidas en su totalidad al usuario.

Las interfaces son la puerta de entrada a los datos, a los resultados, al mundo físico del hardware que, si no se logra, quedará demostrado la discrepancia entre los que los usuarios esperaban que fuera "un acceso a la información rápida, fácil, entretenida y de suma satisfacción para su 
conocimiento y/o aprendizaje" (KAFURE 2009a) contra la realidad. De ahí la necesidad de incrementar esfuerzo en el desarrollo de estas interfaces y de enriquecer el valor emocional de los usuarios fortaleciendo el raciocinio, la atención, la motivación entre otros aspectos psicológicos del usuario.

En la actualidad, en esta era de conectividad urge llegar al usuario donde y como él quiere, es necesario generar nuevo software integrando las más variadas técnicas de diseño.

Tradicionalmente se afirmaba que era necesario evitar la intervención de las emociones, para que la toma de decisiones tuviese éxito. Era una perspectiva muy difundida la de disociar la emoción de la razón, tanto en términos mentales como neurológicos. Actualmente, los avances de la ciencia en el conocimiento del cerebro y sobre cómo la emoción y la cognición se entrelazan, nos comunican que la emoción nos ayuda a escoger entre diversas opciones y posibilidades, en complemento con el conocimiento y la razón (Damásio, 1994, 2005; Norman, 2004). Janczura (1999), las investigaciones de psicología cognitiva, aclara que los usuarios de la información tienen muchas diferencias que merecen ser estudiadas con la finalidad de crear recursos electrónicos y mecanismos para acceder a la información, Hoy en día es vital el deseo y necesidad del individuo para aceptar la información de la mejor manera posible con características visuales de preferencia.

En la interacción humano-computador, puede entenderse por "diseño deseado", las múltiples maneras de sentir, visualizar y comunicarse con la interfaz por medio de diferentes tipos de diseños de despliegues de pantalla. Cada forma de interacción proporciona y permite crear principios de diseño particulares, por ejemplo, lenguaje natural, lenguaje de comando, menú de selección, manipulación directa, entre otros. 
La dificultad o facilidad en el conocimiento de un objeto influyen nuestra percepción del mismo. Es muy posible que no desarrollemos uniones emocionales positivas con algo de difícil uso. Es importante entender que hay consecuencias positivas cuando son utilizadas las interfaces de manera divertida. El mayor desafío en el proceso creativo de desarrollo de las interfaces es, introducir de manera sistemática los requisitos no utilitarios y combinarlos con los requisitos objetivos, para contraponer los estudios de usuarios tradicionales, que suelen centrarse en el comportamiento racional, dejando de lado el comportamiento emocional (Cañada, 2005; Monk et al., 2002; HassanMontero y Martín-Fernández, 2003). Sugimoto et al. (1995) observaba que la usabilidad del Sistema de Gestión de Información es importante porque los sistemas de información de las organizaciones son obligatoriamente utilizados tanto por usuarios con experiencia, como por neófitos. Estas tecnologías avanzadas de hardware y software han traído nuevos y mejores conceptos para construir sistemas de información con usabilidad, tales como: interfaces gráficas de usuarios (GUI), interfaces multimedia y herramientas de soporte colaborativo, entre otras.

La interfaz del SGI permite que los usuarios interactúen instantáneamente con las imágenes y se debe incluso invitar a estos en la concepción de las mismas interfaces.

De acuerdo con Palmer (apud Nöth, 1995), una representación es algo que está en el lugar de otra cosa, es decir, es algún tipo de modelo de algo que representa. Esta teoría implica e integra la existencia de dos mundos que tienen una relación pero funcionalmente separados: el mundo representado y el mundo representante.

Los modelos mentales representan el conocimiento del individuo y su organización. Norman (1983) establece la diferencia de tres tipos de modelos: el modelo mental del usuario, el modelo conceptual del analista, que en este caso también podría ser el del profesional de la información, y el modelo de 
la imagen del sistema. Aquí el modelo mental es la representación mental que el usuario informático se hace de su tarea y de su ambiente de trabajo; el modelo conceptual es la representación que el diseñador (sea este el analista o el profesional de la información) hace de los sistemas, y la imagen del sistema contempla desde la imagen de la interfaz hasta los aspectos físicos y dispositivos de comunicación (figura 1).

Figura 1. Teoría de la acción de Norman: tipos de modelos Fuente: Norman (1983).

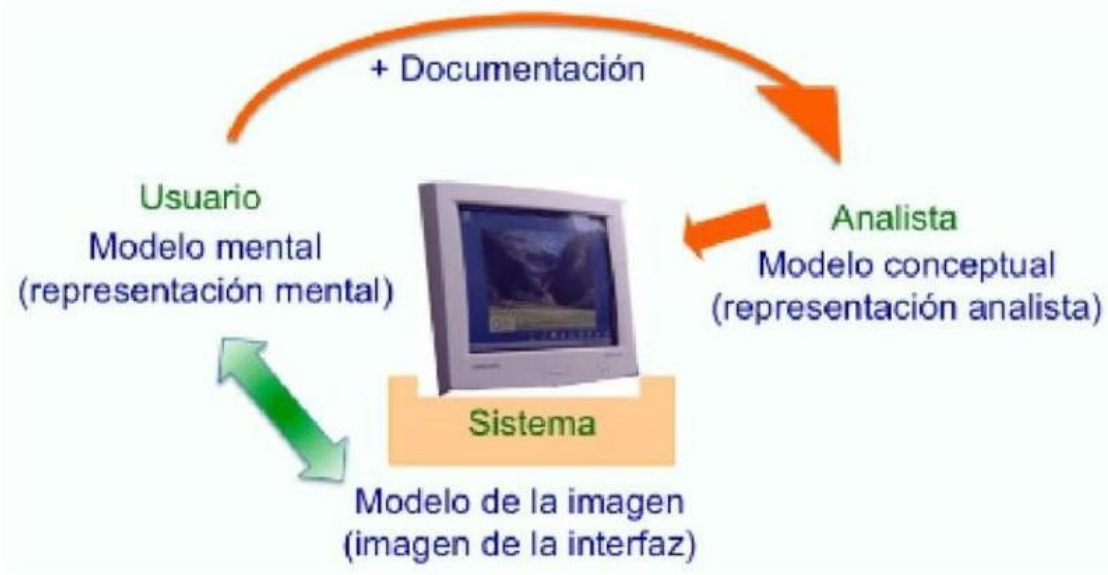

La calidad, versatilidad y variedad de la comunicación entre el usuario y la interfaz depende y pertenece en gran medida de la compatibilidad entre el modelo mental del usuario y la imagen del sistema. Cuanto mayor sea la compatibilidad entre la representación mental del usuario y la imagen de la interfaz, mejor será el entendimiento de la información y por lo tanto, mayores la usabilidad y el diseño emocional de la interfaz (Norman, 1986, 2004; Lula, 1992; Haan, 2000).

Para obtener o adaptar una imagen de interfaz al modelo mental de los usuarios (tabla 1, modelo mental), se hace referencia a este cuadro que tiene como información principal las representaciones internas que los usuarios tienen de la tarea y la actividad: 
Tabla 1. Modelo mental

Necesidades

- Acceso a la información

- Localización rápida de la información buscada

Expectativas

- Aprendizaje fácil de la interfaz del SGI

- Uso fácil de la interfaz del SGI

- Una satisfacción en la interacción usuario-sistema información -institución

Fuente: Adaptada de Kafure (2004, p. 4)

Existen grandes y valiosos documentos que hablan de la robótica y sus interfaces, estos documentos tratan de la integración de los organismos robóticos con varios lenguajes de programación entre ellos el de Vivet, algunos de los fundadores de la robótica educativa como es la obra de Papert, Saymour, Mindstorm, Children, Computers an power fuel ideas. Brighton, HervesterPress, 1980 y The Cildren's Machine: rethinking School in the age of the computer. Basic Boock, New York. 1992 así como las memorias de Vivet Martial plenamente referidos en las memorias de los congresos internacionales de robótica en Bélgica de 1993. Si hacemos un estudio en la actualidad podríamos descubrir como insignia en el mercado marcas que han demostrado robustez tecnológica y grandes desarrollos de interfaces usuarios-origen(Hardware) como son: los robots de LEGO, Fischerrechnik, Mecano entre otras, que nos dicen que es muy posible y fácil dar vida a organismos robóticos gracias a la existencia de interfaces y lenguajes de programación embebidos que hacen posible el entendimiento del usuario con lo que está apunto de crear y luego visualizar como 
resultado de su acción, tanto ordenes de entrada así, como respuestas de estos organismos robóticos en sus siguientes lecturas de sensores.

\section{Discusión}

Se han superados los paradigmas que proyectaba la organización y la recuperación de la información con estructuras de flujos de datos bastante homogéneas y lineales que usaban solamente texto, imágenes, sonidos y en menor escalas animaciones.

Las interfaces de sistemas de información robótica son el conjunto de actividades que en algunos casos pueden ser pedagógicas, que apoyan y fortalecen áreas específicas del conocimiento y desarrollan competencias en el alumno a través de la visualización, facilidad y sencillez, permitiendo la concepción, creación, ensamble y puesta en funcionamiento de robots.

El objetivo de incluir sistemas de información amigables en la enseñanza de la robótica, es lograr una adaptación de los alumnos a los procesos productivos actuales, en donde la automatización (Tecnología que está relacionada con el empleo de sistemas mecánicos, electrónicos y basados en computadoras; en la operación y control de la producción) juega un rol muy importante. Cabe recalcar que la robótica se considera un sistema que va más allá de una aplicación laboral pero una vez aplicada es vital brindar al usuario herramientas que favorezca la toma de decisiones de forma rápida y sin complicaciones de lectura homogénea.

Es por lo tanto, esencial mencionar que en el estudio de la robótica, existe una gran necesidad de una perfecta relación entre el software y el hardware, ya que los movimientos que realizará un robot son acoplamientos y comparticiones de información entre lo físico y lo lógico. 
Las interfaces de sistemas de información tienen un valor estratégico y deben estar disponibles a cualquier hora y en cualquier lugar, y deben ser mejoradas con la integración usuario-diseñador con actividades intelectuales colaborativas. Es preciso dar un soporte fundamentado y lógico en el diseño emocional y en la usabilidad, e invitar a los usuarios a que sean co-autores para interactuar y construir universos virtuales en un intercambio, tanto de ideas, necesidades, así como en el posicionamiento de responsabilidades.

Para concebir, enriquecer y adaptar los recursos electrónicos y los mecanismos de búsqueda de la información al deseo, y facilidad de los individuos, con el diseño emocional y usabilidad, es indispensable tener en cuenta la participación de los usuarios, aprender más sobre sus requerimientos y considerar sus limitaciones de absorción de la misma información de los datos; por ejemplo, puede observarse, que al usuario le lleva más tiempo "conocer" la imagen complicada de la interfaz, visualizada en la pantalla del computador, que una caricatura de tres trazos a lápiz. A priori, sería absurdo admitir la posibilidad de que un usuario (mecanismo psicofisiológico) pudiese adquirir instantáneamente una cantidad ilimitada de información. O en otro ejemplo es difícil creer que el usuario pueda normalmente percibir de manera instantánea todo un mapa geográfico en sus más mínimos detalles o aprender una página de enciclopedia con tan solo una mirada. Según Moles (1969) para evitar esos problemas, podría medirse la pérdida relativa de la transmisión de la información a través del canal información (la interfaz en este caso): cuanto más eficiente, eficaz y satisfactorio el canal de comunicación, más cantidad de información transmitida es recuperada por los usuarios.

El diseño de la interfaz la convierte en un instrumento importante que hace parte de un ciclo pequeño que comanda soberanamente el ciclo mayor: el de la interacción humano-computador. En esta concepción debe existir compatibilidad entre la interfaz y la comprensión del individuo sin 
obviar la armonía. La armonía se convierte en el presupuesto para operaciones rápidas y precisas; reduce el riesgo de fallas, incidentes y errores en la realización de la tarea (Grandjean, 1998).

Las interfaces tienen como objetivo de proporcionar, convertir y comunicar, de forma general, una o varias de las siguientes funciones:

- Entradas analógicas, que convierten niveles analógicos de voltaje o de corriente en información digital procesable por el ordenador. A este tipo de entradas se pueden conectar distintos sensores analógicos, como por ejemplo una LDR (resistencia dependiente de la luz).

- Salidas analógicas, que convierten la información digital en corriente o voltaje analógicos de forma que el ordenador pueda controlar sucesos del "mundo real". Su principal misión es operar distintos equipamientos de control: válvulas, motores, servomecanismos, etc.

- $\quad$ Entradas y salidas digitales, usadas en aplicaciones donde el sistema de control solo necesita discriminar el estado de una magnitud digital (por ejemplo, un sensor de contacto) y decidir la actuación o no de un elemento en un determinado proceso, por ejemplo, la activación / desactivación de una electroválvula.

- $\quad$ Recuento y temporización, algunas tarjetas incluyen este tipo de circuitos que resultan útiles en el recuento de sucesos, la medida de frecuencia y amplitud de pulsos, la generación de señales y pulsos de onda cuadrada, y para la captación de señales en el momento preciso.

Los robots son máquinas cada vez más complejas que para poder programarlos y controlarlos se hace necesario un conocimiento técnico. En la mayoría de los casos, cuantas mayores capacidades posee un robot, mayor es la complejidad de su diseño e igual complejidad para su control. Además, la mayoría de los robots no poseen interfaces simples para poder utilizarlos. Esto hace también que 
las interfaces suelen ser complejas involucrando lenguajes de programación para explotar de mejor manera su funcionalidad, planteando un problema para el uso de los robots por parte del público inexperto.

En los últimos años, el desarrollo plataformas y entornos de programación de robots desempeña un papel importante en la investigación en robótica en general maximizando el uso en particular, en actividades vinculadas a la Robótica Educativa.

Existen varios desarrollos de modelos comerciales de interfaces gráficas, entre los que se pueden mencionar:

- Robot Educativo Denso, de DeROBÓTICA

- Interfaz FlowGo, de Data Harvest

- $\quad$ Ladrillo RCX, de Lego

- $\quad$ Interfaz ROBO TX Controller de fischertechnik

- Interfaz Enconor, de Enconor Tecnología Educativa

- $\quad$ Robot Programable Moway, de Minirobots Sistema constructivo Multiplo.

Esta variedad de diseños y elementos llevan a la creación no solo de interfaces sino de kits de robots de enseñanza e introducción a la programación:

- $\quad \operatorname{mBot}$ (Arduino) (software y hardware de desarrollo libre)

\section{- $\quad$ LEGO MINDSTORMS NXT}

- $\quad$ Printocho, un robot para enseñar a programar a los niños con Arduino 


\section{- Kibo}

- $\quad$ Vortex (Arduino)

- $\quad$ Poppy (Arduino)

- Zowi, el Robot de Clan

- $\quad$ Kit de robótica de BQ (Arduino)

- $\quad$ Dash \& Dot vs Ollie.

\section{Conclusión}

De manera general, los analistas y desarrolladores de sistemas esperan que los usuarios procedan de acuerdo al modo como la interfaz funciona, pero direccionada y fundamentada en la noción de modelo mental que ellos visualicen y expresen, es decir un modelo propio. En este modelo, el individuo construye una representación mental de sus objetivos (tarea) y del ambiente con el cual interactúa. De esta posición que asumía que era el usuario quien debía adaptarse a la interfaz y trabajar con limitaciones, se ha ido pasando al concepto de que sea la interfaz la que se adapte al usuario, por medio de una facilidad de uso. Podemos deducir que cuanto más se conozca al usuario y a los datos que estas deben mostrar, más adaptada podrá ser la imagen de la interfaz a su modelo requerido y entendible para ellos.

Facilitar el acceso a los datos y a la información por medio de un soporte material, como la interfaz de un sistema de información, es parte del direccionamiento que debe considerar no sólo la objetividad sino también la subjetividad de los usuarios, este direccionamiento había sido relegado por los modelos teóricos representantes de la ciencia cognitiva. Es inherente recordar cómo Carl 
Gustav Jung (apud Arnheim, 1989) se sorprendía con frecuencia al constatar que los seres humanos somos incapaces de comprender un punto de vista diferente al propio y admitir su validez.

El usuario de los sistemas es una persona que tiene la posibilidad de solicitar servicios de información para cumplir con responsabilidades, así como de acrecentar sus capacidades profesionales para brindar comodidades familiares a su comunidad o también a estudiantes con deseo de aprender aquellas herramientas que en el futuro le permitirán el desarrollo de interfaces. Esta información puede solicitarse por medio de interfaces de sistemas de gestión de la información diseñadas para el usuario ya la vez facilitar el logro de sus objetivos.

El hecho de evaluar los proyectos de interfaces desde sus inicios o al menos en sus primeras etapas contribuye a reducir las diferencias entre el modelo mental de los usuarios (tabla 1) y el modelo conceptual de los analistas y profesionales de la información.

Se ha investigado cómo diseñar y organizar la información acorde a los requerimientos (modelo mental), la tarea y la actividad de los usuarios. Este proceso ha permitido identificar y comprender los aspectos cognitivos y los emocionales en el vínculo existente entre la imagen de la interfaz y la representación mental del usuario en base a las respuestas que pueda dar un robot con sus elementos. Se hizo posible verificar las estrategias imaginativas, en las que se unen la emoción, el conocimiento y la razón, que las personas, cuando están más concentradas, relajadas y felices, pueden abrir un espacio para que sus procesos de raciocinio se expandan, y dar cabida a la creatividad y a la imaginación y estas a su vez al desarrollo eficiente de software.

Es así que un número considerable de publicaciones agrupan excelentes recomendaciones para agregar valor a la interfaz del usuario después de ser evaluada. 


\section{Existen varios tipos:}

- Guías de recomendaciones: son publicaciones que agrupan recomendaciones genéricas derivadas y validadas empíricamente

- Normas: son documentos oficiales disponibles públicamente que ofrecen requisitos para el proyecto (y evaluación) de la interacción. La norma internacional ISO 20282 presenta el término “facilidad de operación”, relacionado con el concepto de la usabilidad, preocupado y direccionado hacia la interfaz del usuario de productos de uso cotidiano, teniendo en cuenta las características del usuario. Entre otras tenemos la norma internacional ISO 9241 - Requisitos ergonómicos para trabajo de escritorio con terminales de video, está dividida en 17 partes.

- Guías de estilo: son documentos normalmente producidos por una organización y facilitados comercialmente. Ejemplos: SUN Microsoft, OSF/Motif, OPEN LOOK, IBM CUA, e User Interface Guidelines / Apple.

Se han hecho recomendaciones sobre cómo integrar a los usuarios y a la lógica de los analistas, trayendo beneficios y sumando valor a la interfaz de los sistemas de información con Robótica. Se ha producido un aumento en la cualidad de la usabilidad y el diseño emocional en la recuperación de la información, mucho antes de que las interfaces sean distribuidas y utilizadas formalmente, logrando disminuir la distancia entre las necesidades y expectativas de los usuarios y su funcionalidad. Después de hacer la evaluación del uso y el diseño de las interfaces, los usuarios aceptan y expresan que la interacción es más agradable, satisfactoria y rápida.

\section{Bibliografía.}

Arnheim, R. 1989. Intuição e intelecto na arte. São Paulo: Martins Fontes. 
Cañada, J. 2005. Entrevista sobre los paradigmas del diseño. Diseño emocional: definición, metodología y aplicaciones. Santiago de Chile: Universidad Tecnológica Metropolitana. Tesis (Maestría)

Cañas, J.J. y Waerns, Y. 2001. Ergonomía cognitiva, aspectos psicológicos de la interacción de las personas con la tecnología de la información. Madrid: Médica Panamericana.

Costa, P. de J. F. y Scavardoni, P. E. B. 2009. Aumento da usabilidade de ferramentas de avaliação de interfaces humano-computador. WICI'2009, Workshop Internacional em Ciência da Informação: inovação tecnológica e preservação da informação. Brasília: Universidade de Brasília.

Damásio, A.R. 1994. Descartes' error: emotion, reason, and the human brain. New York: Grosset/ Putnam.

Damásio, A.R. 2005. Somos esclavos de las emociones y del entorno. El País [en línea] 2005, Oct. 21. [citado octubre 6, 2009] Disponible en http://www.elpais.com/articulo/sociedad/Somos/esclavos/emociones/entorno/elpporsoc/2005 $\underline{1021 \text { elpepisoc_9/Tes }}$

Duarte, G. de M. y ALVES, L. de O. 2009. Ferramenta de avaliação de Sistemas Informatizados de Gestão Arquivística de Documentos (SIGAD). En: WICI 2009 - Workshop Internacional em Ciência da Informação: Inovação tecnológica e preservação da informação. Brasília: Universidade de Brasília.

Frost, C.O. 2001. The role of mental models in a multi-modal image search. Proceedings of the ASIST Annual Meeting, 2001, vol. 38, p. 52-57.

Grandjean, E. 1998. Manual de ergonomia: adaptando o trabalho ao homem. Porto Alegre: Bookman. GUÉRIN, F. et al. 2001. Compreender o trabalho para transformá-lo: a prática da ergonomia. São Paulo: Editora Edgar Blücher.

Haan, G. de. 2000. A formal model of competence knowledge for user interface desing. Amsterdam: Vrije Universiteit. Tesis (Doctorado)

Hassan-Montero, Y., y Martín-Fernández, F.J. 2003. Más allá de la usabilidad: interfaces 'afectivas'. No Solo Usabilidad [en línea] octubre 20, 2003 [citado octubre 6, 2009] Disponible en: http://www.nosolousabilidad.com/articulos/interfaces_afectivas.htm

Janczura, G. A. 1999. Acessibilidade conceitual em tarefas de categorização e memória. Brasília: Universidade de Brasília: CNPq.

Johnson-Laird, P. y Byrne, R. 2000. Mental models website: a gentle introduction. [en línea] [citado octubre 6, 2009] Disponible en Web: http://www.tcd.ie/Psychology/Ruth_Byrne/mental_models/

Kafure, I. 2004. Usabilidade da imagem na recuperação da informação no catálogo público de acesso em linha. Brasília: Departamento de Ciência da Informação e Documentação, Universidade de Brasí- lia. Tesis (Doctorado)

Kafure, I., et al. 2007. Evaluación de la usabilidad de la biblioteca digital PREDICA. En: Seminário Internacional de Bibliotecas Digitais Brasil. Sep. 18-20 2007, Sao Paulo, Bra.

Kafure, I. 2009a. Evaluación de la usabilidad en interfaces de sistemas de gestión documental. En: 2o Congreso Internacional de Investigación en Ciencia de la Información. Medellín: Universidad de Antioquia.

Kafure, I. 2009b. Usabilidad y diseño emocional en la gestión de la información. En: Congresso Internacional de Interacción Persona-Ordenador (Interacción 2009). 
Kafure, I. Y Marzano, A. P. T. 2009. Usabilidade e design emocional na gestão arquivística de documentos escolares. III Seminário de Educação Infantil. Brasília: Associação Pró Educação Vivendo e Aprendendo.

Kafure, I. 2010. Projeto: Design emocional na otimização de recursos informacionais escolares. Brasília: Departamento de Ciência da Informação e Documentação, Universidade de Brasília.

Löbach, B. 2001. Design industrial: Bases para a configuração dos produtos industriais. São Paulo: Edgar Blücher. LULA, B. Jr. 1992. Elaboration d'un environnement de génération interactive d'interfaces à manipulation directer pour le language

Objlog. Tesis (Doctorado), França: Universidade de Droit d'Economie et des Sciences d'Aix-Marseille III, Faculté des Sciences et Techniques de Saint - Jérôme.

Mayer, R. 2005. Cognitive theory of multimedia learning. En: R. Mayer, The Cambridge handbook of multimedia learning. NY: Cambridge University Press. p. 31-48.

Moles, A. 1969. Teoria da informação e percepção estética. Rio de Janeiro: Tempo Brasileiro. MONK, A., et al. 2002. Funology: Designing enyoyment. Conference extended abstracts on human factors in computer systems. Minneapolis, Minnesota. p. 924-925.

Norman, D. A. 1983. Some observations on mental models. En: D. Gentner y A.L. Stevens, Mental models. Hillsdale, New Jersey: Lawrence Erlbaum Associates. p. 7-14.

Norman, D. A. 1986. Cognitive engineering. En: User centered system desing: New perspectives on humancomputer interaction. Hillsdale, NJ: Erlbaum Associates.

Norman, D. A. 2004. Emotional design: why we love (or hate) everyday things. New York: Basic Books.

Nöth, W. 1995. Panorama da semiótica: de Platão a Peirce. São Paulo: Annablume.

Pressman, R.S. 2005. Ingeniería del software, un enfoque práctico. Madrid: McGrawHill.

Sebillote, S. 1995. Task TD-5: methodology guide to task analysis with the goal of extracting relevant characteristics for interfaces, Esprit 3 Projet: P6593, "INTUITIVE". INRIA Rocquencourt, France.

Shneiderman, B. 1998. Designing the user interface - strategies for effective human-computer interaction. Addinson Wesley Publishing Co. STERNBERG, R. J. 2000. Psicologia cognitiva. Porto Alegre: Artes Médicas.

Sugimoto, S., et al. 1995. Enhancing usability of network-based library information system - experimental studies of a user interface for OPAC and of a collaboration tool for library services. Digital libraries ' 95 . [en línea]1995[citado octubre 6, 2009] Disponible en web: http://www.csdl.tamu.edu/ DL95/papers/sugimoto/sugimoto.html 